\title{
Spherical Light Fields
}

\section{Bernd Krolla ${ }^{1}$}

http://av.dfki.de/ krolla

Maximilian Diebold ${ }^{2}$

http://hci.iwr.uni-heidelberg.de/Staff/mdiebold/

Bastian Goldlücke ${ }^{3}$

http://www.informatik.uni-konstanz.de/cvia/

Didier Stricker ${ }^{1}$

http://av.dfki.de/stricker

\section{${ }^{1}$ German Research Center for Artificial Intelligence, Kaiserslautern, Germany \\ ${ }^{2}$ Heidelberg Collaboratory for Image Processing, Heidelberg, Germany \\ ${ }^{3}$ Department for Computer and Information Science, University of Konstanz, Konstanz, Germany}

A full view spherical camera exploits its extended field of view (FOV) to map its complete environment onto a $2 \mathrm{D}$ image plane. Thus, with a single shot, it delivers a lot more information about the surroundings than one can gather with a normal perspective or plenoptic camera, which are commonly used in light field imaging. However, in contrast to a light field camera, a spherical camera does not capture directional information about the incident light, and thus a single shot from a spherical camera is not sufficient to reconstruct 3D scene geometry.

In this paper, we introduce a method combining spherical imaging with the light field approach. To obtain 3D information with a spherical camera, we capture several independent spherical images by applying a constant vertical offset between the camera positions and combine the images in a Spherical Light Field (SLF).

Our approach differs from its related work in terms of expanded FOV and reduced acquisition time: Taguchi et al. [2] used an array of spherical mirrors to model catadioptric cameras for wide angle light field rendering, which implies decreasing tangential resolution close to the mirror borders and limits the FOV to $150^{\circ} \times 150^{\circ}$. Unger et al. [4] employed a fisheye-camera translated on a plane to capture hemispherical HDR images of a scene. The total acquisition time of up to 12 hours for a single scene restricts the application scenario to constantly illuminated indoor environments. Our proposed approach for SLF acquisition uses spherical cameras as shown in Figure 1(a) and allows to capture scenes within a few minutes, making it applicable to outdoor scenes.

A convenient description of this camera type is provided by Torii $e t$ al. [3], who consider a spherical camera to consist of a camera center $C$ with a surrounding unit sphere acting as projection surface. This definition implies that no intrinsic parameters such as focal length or distortion values known from perspective imaging need to be considered (Figure 1(b)). By applying the Mercator projection [1], the spherical image is conformally mapped to an image on a cylinder surface $\Pi$ (Figure 1(c)) allowing for epipolar plane image (EPI) reconstruction.

To describe a SLF, we define a new parametrization for the camera domain and the surrounding spherical 2D mapped image. We take the cylinder surface $\Pi$ and denote the center line with $\Omega$. The cylinder surface $\Pi$ is parametrized by the image coordinates $(\phi, \theta) \in \Pi$. The line $\Omega$ contains the focal points $t \in \Omega$ of all possible camera positions in vertical direction.

A Spherical Light Field can then be described by a function

$$
L: \Omega \times \Pi \rightarrow \mathbb{R} \quad(t, \phi, \theta) \mapsto L(t, \phi, \theta),
$$

where $L(t, \phi, \theta)$ defines the intensity of the incident light ray on the image plane $(\phi, \theta)$ passing through the focal point $t$. To estimate the disparity, we address a 2D slice $\Sigma_{\phi^{*}}$ of the SLF by setting $\phi$ to a fixed value $\phi^{*}$. The restriction of the light field to such a slice defines an EPI, being formally given as

$$
\begin{array}{r}
S_{\phi^{*}}: \Sigma_{\phi^{*}} \rightarrow \mathbb{R} \\
(\theta, t) \mapsto S_{\phi^{*}}(\theta, t):=L\left(t, \phi^{*}, \theta\right) .
\end{array}
$$

Assuming a Lambertian scene, the EPI yields information about the disparity of a scene point in the form of orientated lines. To compute the disparity on the EPI, we can thus perform an orientation analysis on the given EPI $S_{\phi^{*}}$, using a structure tensor. The orientation angle and thus the disparity map for the EPI $S_{\phi^{*}}$ can be computed directly from the components of the structure tensor.

A example for a resulting disparity map, is shown in Figure 1(d) and was computed by iterating over all EPIs from the SLF and storing the computed disparity at the corresponding azimuthal slice.
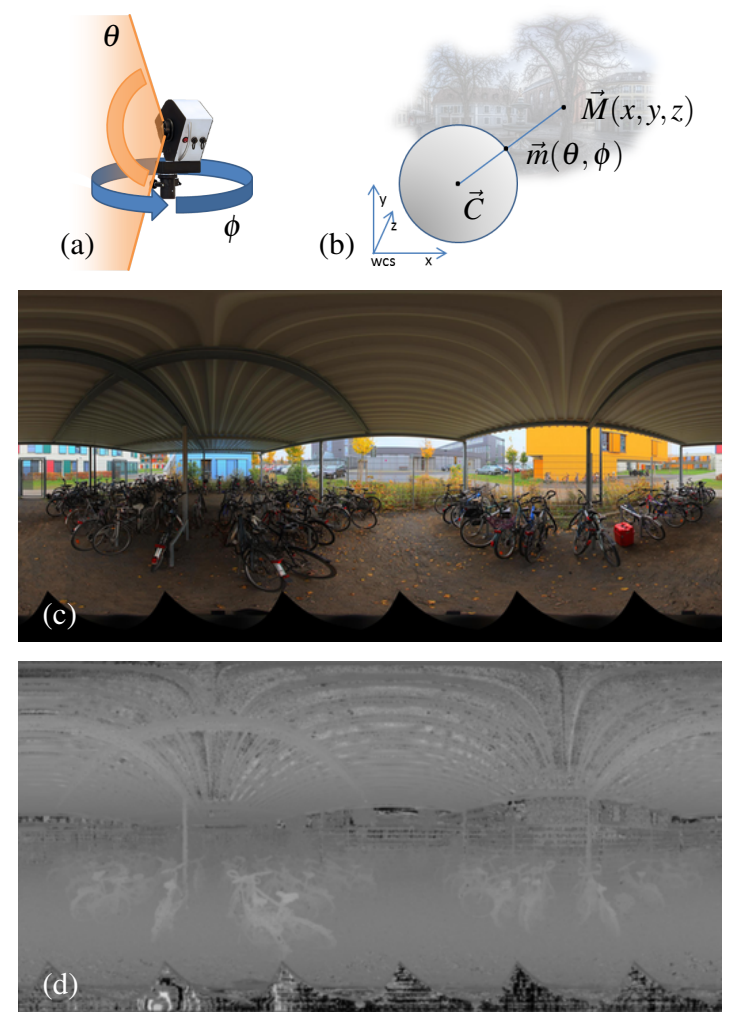

Figure 1: (a) Spherical image acquisition using a rotating tripod mounted camera equipped with a fish eye lens. (b) The spherical image results from the back projection of 3D points $M(x, y, z)$ to their corresponding image points $m(\theta, \phi)$ with $\phi[0,2 \pi)$ and $\theta[0, \pi]$ assuming $C$ to be the cameras center of projection. (c) In the current work, the resulting image is a High Dynamic Range (HDR) image with a resolution of $14000 \times 7000$ pixel. (d) shows the resulting disparity map of the captured scene.

The resulting full view spherical disparity map can then be employed for a 3D scene reconstruction of the camera's surroundings. Benchmarks on synthetic datasets demonstrate good accordance with the ground truth data. Finally simplifies the combination of spherical and HDR imaging approaches greatly the task of disparity estimation for real scenes, e.g. due to improved contrast, as shown in our work.

[1] Mats Bentsen, Geir Evensen, Helge Drange, and Alistair Jenkins. Coordinate transformation on a sphere using conformal mapping. $M W$ Review, (127):2733-2740, 1999.

[2] Yuichi Taguchi, Amit Agrawal, Ashok Veeraraghavan, Srikumar Ramalingam, and Ramesh Raskar. Axial-cones: Modeling spherical catadioptric cameras for wide-angle light field rendering. ACM Transactions on Graphics-TOG, 29(6):172, 2010.

[3] Akihiko Torii, Atsushi Imiya, and Naoya Ohnishi. Two- and threeview geometry for spherical cameras. In Proceedings of the sixth workshop on omnidirectional vision, camera networks and nonclassical cameras. Citeseer, 2005.

[4] Jonas Unger, Andreas Wenger, Tim Hawkins, Andrew Gardner, and Paul Debevec. Capturing and rendering with incident light fields. In Proceedings of the 14th Eurographics workshop on Rendering, pages 141-149. Eurographics Association, 2003. 